\title{
Editorial
}

\section{Uncertainty Analysis in Reactor Physics Modeling}

\author{
Kostadin Ivanov, ${ }^{1}$ Carlo Parisi, ${ }^{2}$ and Oscar Cabellos ${ }^{3}$ \\ ${ }^{1}$ Pennsylvania State University, University Park, PA 16802, USA \\ ${ }^{2}$ ENEA, Santa Maria di Galeria, 00123 Rome, Italy \\ ${ }^{3}$ Universidad Politecnica de Madrid, 28006 Madrid, Spain \\ Correspondence should be addressed to Kostadin Ivanov; knil@psu.edu
}

Received 18 November 2013; Accepted 18 November 2013

Copyright @ 2013 Kostadin Ivanov et al. This is an open access article distributed under the Creative Commons Attribution License, which permits unrestricted use, distribution, and reproduction in any medium, provided the original work is properly cited.

In recent years, there has been an increasing demand from nuclear research, industry, safety, and regulation for best estimate predictions to be provided with their confidence bounds. Consequently, Organization for Economic Cooperation and Development (OECD)/Nuclear Energy Agency (NEA) has initiated an international uncertainty analysis in modeling (UAM) benchmark focused on uncertainty analysis in best-estimate coupled code calculations for design, operation, and safety analysis of light water reactors (LWRs). The title of this benchmark is "OECD/NEA UAM-LWR benchmark". Reference systems and scenarios for coupled code analysis are defined to study the uncertainty effects for all stages of the system calculations. Measured data from plant operation are available for the chosen scenarios.

The proposed technical approach is to establish a benchmark for uncertainty analysis in best-estimate modeling and coupled multiphysics and multiscale LWR analysis, using as bases a series of well-defined problems with complete sets of input specifications and reference experimental data. The objective is to determine the uncertainty in LWR system calculations at all stages of a coupled reactor physics/thermal hydraulics calculation. The full chain of uncertainty propagation from basic data, engineering uncertainties, across different scales (multi-scale), and physics phenomena (multiphysics) is tested on a number of benchmark exercises for which experimental data are available and for which the power plant details have been released. The principal idea is (a) to subdivide the complex system/scenario into several steps or exercises, each of which can contribute to the total uncertainty of the final coupled system calculation, (b) to identify input, output, and assumptions for each step, (c) to calculate the resulting uncertainty in each step and (d) to propagate the uncertainties in an integral system simulation for which high quality plant experimental data exist for the total assessment of the overall computer code uncertainty. The main scope covers uncertainty (and sensitivity) analysis (SA/UA) in best estimate modeling for design and operation of LWRs, including methods that are used for safety evaluations. As part of this effort, the development and assessment of different methods or techniques to account for the uncertainties in the calculations are to be investigated and reported to the participants.

The general frame of the OECD/NEA UAM-LWR benchmark consists of three phases with different exercises for each phase: Phase I (neutronics phase), Phase II (core phase), and Phase III (system phase). The focus of Phase I is on propagating uncertainties in standalone neutronics calculations and consists of the following three exercises.

Exercise I-1: "Cell Physics" focused on the derivation of the multigroup microscopic cross-section libraries and associated uncertainties.

Exercise I-2: "Lattice Physics" focused on the derivation of the few-group macroscopic cross-section libraries and associated uncertainties.

Exercise I-3: "Core Physics" focused on the core steady state stand-alone neutronics calculations and associated uncertainties.

The special issue is devoted to Phase I of the abovedescribed OECD/NEA UAM-LWR benchmark. The special issue contains thirteen papers devoted to the advances in the uncertainty and sensitivity analysis related to reactor physics modeling. The authors of these papers are active participants 
in the OECD/NEA UAM-LWR benchmark and in this publication they present their work and developments related to the benchmark exercises. Most of the papers are focused on propagation of cross-sections uncertainties at different stages of reactor calculations and some papers also discuss in addition the propagation of manufacturing uncertainties in lattice physics calculations. The topic of the paper of M. Pusa is the development of sensitivity and uncertainty analysis capability for the reactor physics code CASMO-4. The development of two complementary techniques direct perturbation (DP) and stochastic sampling (SS) as well as their implementation in CASMO-5MX is described by W. Wieselquist et al. The development and application of SS technique using MCNPX was also presented in the same paper. The variance-based global sensitivity analysis technique has been modified to be applied to calculations of reactor parameters that are dependent on group-wise neutron cross-sections as reported by B. A. Adetula and P. M. Bokov. Furthermore, P. M. Bokov presented asymptotic analysis for the variance-based global sensitivity indices in a companion paper. L. Mercatali et al. performed sensitivity and uncertainty analysis using SCALE6.1 code package (based on the generalized perturbation theory) in order to assess the prediction uncertainty of some selected reactor integral parameters due to the uncertainty in the basic nuclear data. Moreover, the reference solutions for the test cases of Exercise I-1 obtained using Monte Carlo methodologies together with a comparison between deterministic and stochastic solutions are presented in the same paper. Studies involving criticality and uncertainty analysis calculations on both pin cell and fuel assembly level were performed using TSUNAMI-2D sequence in SCALE 6.1 and subsequently presented by C. C. Arenas et al. Uncertainty variations due to temperatures changes and different fuel compositions were the main focus of this analysis. C. J. Díez et al. reported sensitivity calculations using the "sandwich formula" to propagate cross-section uncertainties. Two different codes were employed to calculate the sensitivity coefficients of $k_{\text {eff }}$ to cross-sections in criticality calculations: MCNPX2.7e (using differential operator technique) and SCALE6.1 (using adjoint-weighted technique). The effects of the numerical discretization error and the manufacturing tolerances on fuel pin lattice integral parameters (multiplication factor and macroscopic cross-sections) through sensitivity calculations were discussed by E. Canuti et al. Evaluation of uncertainties in depletion calculations is the subject of the papers of O. Cabellos and H. J. Park et al., based on the PWR pin-cell burnup subexercise of Exercise I-1. Two methods for propagating cross-section uncertainties through core simulators (the XSUSA statistical approach and the "twostep" method) were compared and analyzed by A. Yankov et al. The paper of C. Mesado et al. studies the propagation of uncertainties of neutron kinetics parameters in the coupled core transient response. G. Strydom summarized the results of an uncertainty and sensitivity quantification investigation performed with statistical methodology, utilizing a typical high temperature reactor benchmark and the coupled code PEBBED-THERMIX.

The obtained results from sensitivity and uncertainty analysis for the test cases of Exercises I-1 and I-2 indicated that the largest contributors to prediction uncertainties of infinite multiplication factor are the neutron capture reaction ${ }^{238} \mathrm{U}(n, \gamma)$ and corresponding cross-section (for UOX fuel) and ${ }^{238} \mathrm{U}\left(n, n^{\prime}\right)$ reaction and corresponding cross-section (for MOX fuel and UOX fuel with $\mathrm{Gd}_{2} \mathrm{O}_{3}$ burnable poison). When depletion is involved for UOX fuel the major contributor ${ }^{238} \mathrm{U}(n, \gamma)$ is replaced by ${ }^{239} \mathrm{Pu}$ nubar (average number of neutrons per fission reaction) as the burnup process progresses (at approximately $15 \mathrm{GWd} / \mathrm{MTU}$ ).

The cross-section uncertainty information is considered as the most important source of input uncertainty for Phase I of the OECD/NEA UAM-LWR benchmark. The crosssection related uncertainties are propagated through the 3 exercises of Phase I. In Exercise I-2 manufacturing and geometry (technological) uncertainties are added to account for them in lattice physics calculations. It was found that the contribution of manufacturing uncertainties to prediction uncertainty of neutronics parameters of interest is generally smaller than the contribution of cross-section uncertainties. New and improved methods have been developed and implemented in several calculation sequences from the participants during benchmark activities related to Phase I of the OECD/NEA UAM-LWR benchmark. Such diversity is important for the progress of the project and is important for the collaboration of the UAM benchmark group with the SCALE team at the Oak Ridge National Laboratory (ORNL), USA. One of the outcomes was the finding that the propagation of cross-section uncertainties, uncertainties of depletion and kinetics parameters, and manufacturing uncertainties in core calculations can be done simultaneously in a practical manner by combining deterministic and statistical uncertainty and sensitivity analysis methodologies.

As the OECD/NEA UAM-LWR activities advance, the intention is to publish similar special issues on the remaining two phases of the benchmark. For more information about the OECD/NEA UAM-LWR benchmark please visit http://www.oecd-nea.org/science/wprs/egrsltb/UAM.

\section{Acknowledgments}

The editors would like to thank the members of the Expert Group on UAM (EGUAM) at OECD/NEA, the members of the UAM-LWR Scientific Board, and Dr. Enrico Sartori, Dr. Jim Gulliford, and Dr. Akifumi Yamaji from OECD/NEA, whose support, help, contributions, and encouragement in establishing and carrying out this benchmark are invaluable. Finally, our thanks go to the many authors and researchers who contributed to the technical success of Phase I of this benchmark.

Kostadin Ivanov Carlo Parisi Oscar Cabellos 


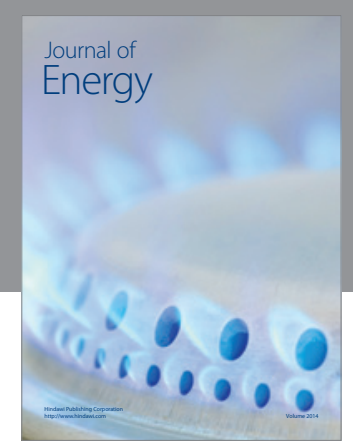

Journal of

Industrial Engineering
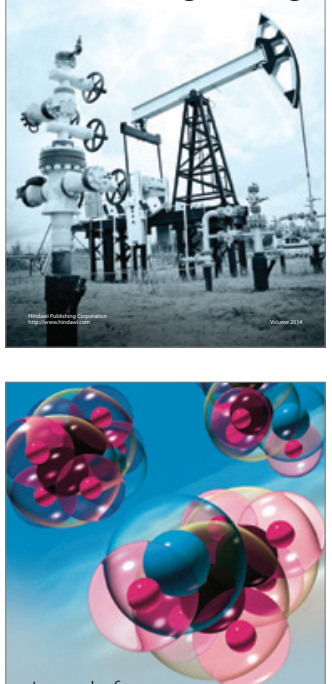

Fuels
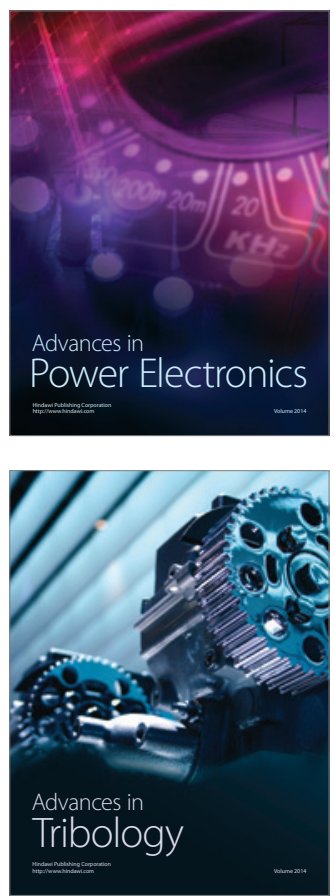

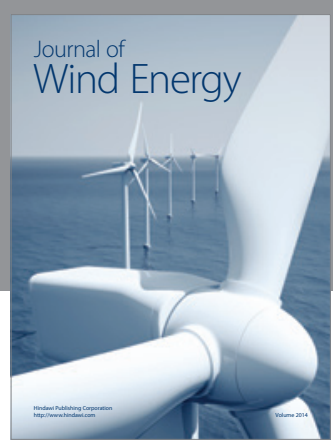

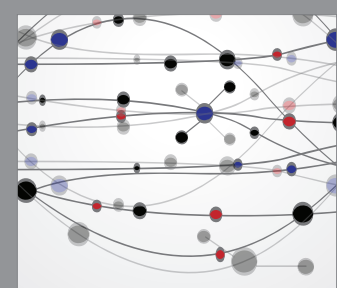

The Scientific World Journal

Submit your manuscripts at http://www.hindawi.com

Journal of

Structures
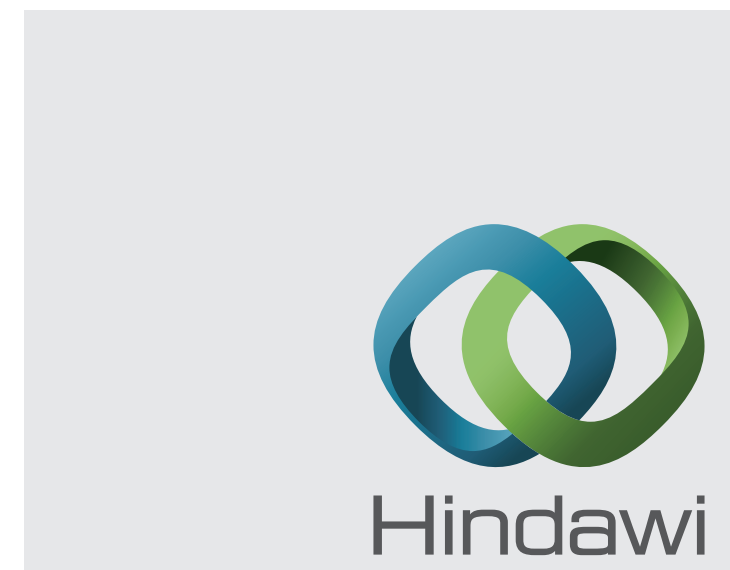

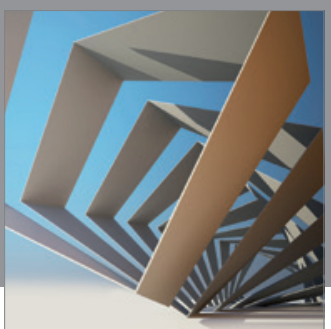

Rotating

Machinery
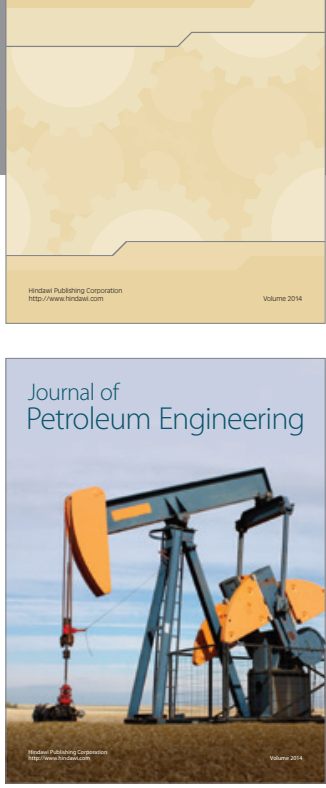

Journal of

Solar Energy
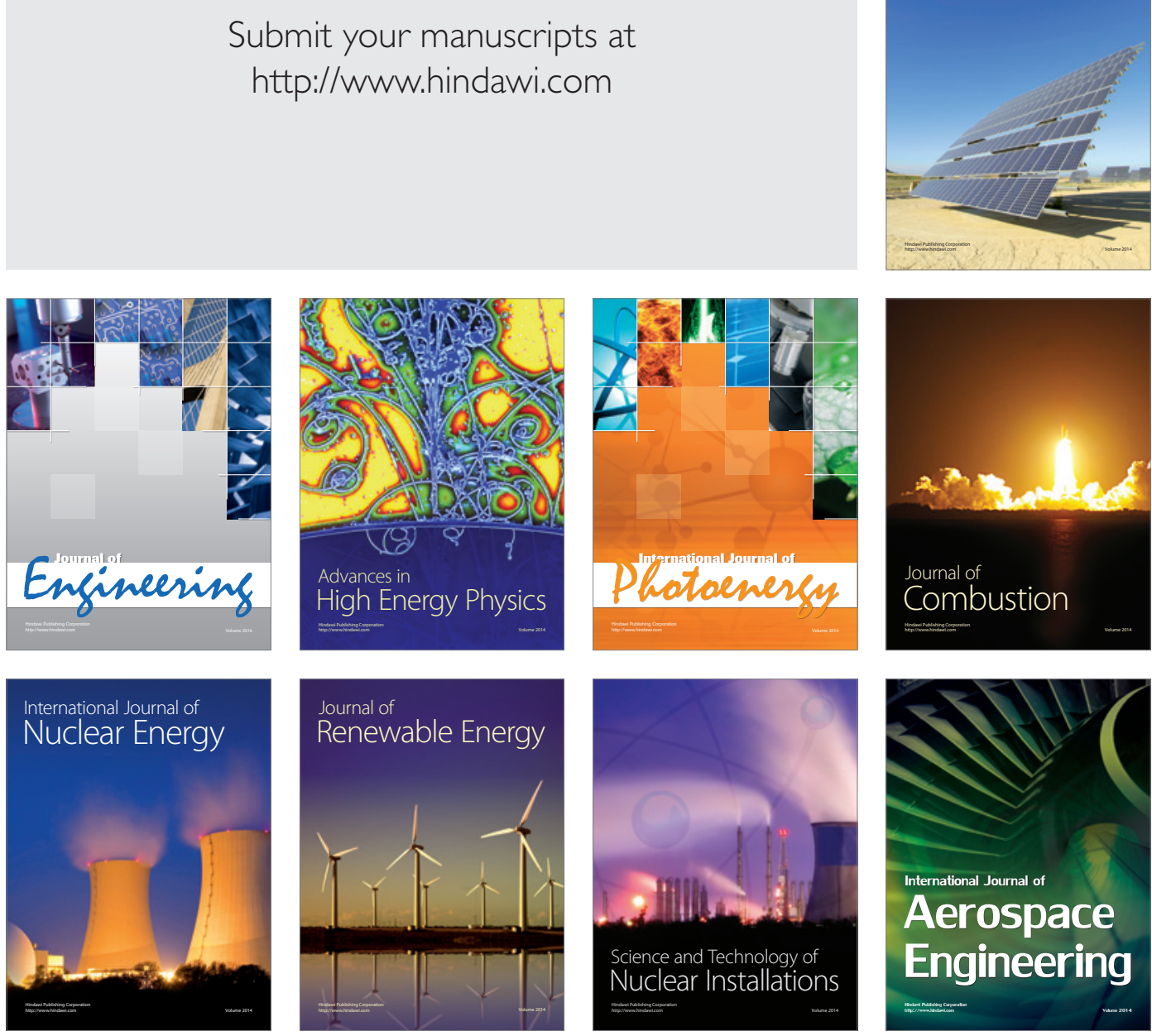